\title{
Pattern Recognition System using MLP Neural Networks
}

\author{
Sarvda Chauhan ${ }^{1}$, Vikas Goel ${ }^{2}$ and Shalini Dhingra ${ }^{3}$ \\ ${ }^{1}$ (EIE Deptt., IET, Bhaddal, Ropar, Punjab INDIA,Punjab INDIA, ${ }^{3}$ (ECE Deptt., IET, Bhaddal, Ropar, Punjab INDIA,
}

\begin{abstract}
Pattern recognition can be used to recognize and/or locate specific objects in an image. The pattern recognition approaches are based on analysis of statistical parameters computed using image processing tools. The parameters may be compared with the standard pattern parameters to identify the pattern or a neural network may be trained using the statistical parameters to identify a given pattern. In the presented work, a neural network approach has been worked out in identifying a pattern. The neural approach applies biological concepts to machines to recognize patterns. A neural network is an information processing system. It consists of massive simple processing units with a high degree of interconnection between each unit. The processing units work cooperatively with each other and achieve massive parallel distributed processing.

The design and function of neural networks simulate some functionality of biological brains and neural systems. The advantages of neural networks are their adaptive-learning, self-organization and fault-tolerance capabilities. For these outstanding capabilities, neural networks are used for pattern recognition applications. In the presented work, multi-layer perceptron (MLP) neural network is used for pattern recognition. Here, each neuron computes a so called "net input" from the outputs of previous neurons and from the weights of the connections. Typically, such a net input is a weighted sum, and a numerical value, called "bias", is added to the net input. In MLPs, a function called "activation function", is applied to the net input. In our experiments, we used a sigmoid function.
\end{abstract}

\section{INTRODUCTION}

Layered neural networks involve a set of input cells connected to a collection of output cells by means of one or more layers of modifiable intermediate connections [1]. The most natural use of this architecture is to implement associatively by associating an input pattern, the representation of a concept or a situation, with another item, either of the same kind or totally different. For this reason, networks with multiple layers are described as associative networks. Pattern recognition is the primary use of this mechanism. Pattern recognition is a popular application in that it enables the full set of human perceptions to be acquired by a machine. In the context of image processing, the different stages are: acquisition, concern with digitizing the data coming from a sensor such as camera or scanner, localizing, which involves extracting the object from its background, and representation. Some features are extracted form the input pattern and that becomes the input to neural network. The final stage, the decision stage, consists of dividing the set of object representations into number of classes. The last two phases, representation and decision, are the associative phases. Layered neural networks can play a part in each of these phases.

\section{BRIEF LiteratURE SURVEY}

Multilayer Feed-forward Neural Networks (MFNNs) is an ideal means of tackling a whole range of difficult tasks in pattern recognition and regression because of its highly adaptable non-linear structure. The trend of using multilayer perceptron neural network [1] for the solution of pattern recognition problem is understandable due to their capacity to accommodate the nature of human brain learning capacity, and the fact that their structure can be formulated mathematically. The functionality of the topology of the MLP is determined by a learning algorithm able to modify the parameters of the net. The algorithm of back propagation, based on the method of steepest descent [4] in the process of upgrading the connection weights, is the most commonly used by the scientific community. Multi-Layer Perceptrons (MLP) are fully connected feed forward nets with one or more layers of nodes between the input and the output nodes. Each layer is composed of one or more artificial neurons in parallel.

\section{FEATURE EXTRACTION}

Feature extraction is a process of studying and deriving useful information from the input patterns. In image recognition, the extracted features contain information about statistical parameters and are computed about the centre of gravity of input pattern. The statistical parameters used in neural network training process are: Perimeter, Area, Maximum and Minimum radii in each quadrant, Intercepts on axes, Mean Radius, Standard Deviation and Figure Aspect. All the parameters discussed here are normalized with respect to the mean radius. The normalization step make the parameters size independent. The statistical parameters so computed above are also made rotation independent using the orthogonal transformation of the coordinates . 


\section{Methodology}

An input pattern is presented to the first stage of the forward paths, the input layer $\mathrm{K}$, which consists of a twodimensional array of receptor cells. The second stage of forward paths is called the hidden layer J. The third stage of the forward paths is the recognition layer I as shown in below figure. After the process of learning ends, the final result of the pattern recognition shows- in the response of the cells of FA. Let $\mathrm{x} 1, \mathrm{x} 2, \ldots, \mathrm{xn}$ be the input signals and w1, w2, w3, ,.., wn be the synaptic weights, and u the activation potential, \& the threshold and y the output signal and $\mathrm{f}$ the activation function:

$$
u=\sum_{i=1}^{N} w_{i} x_{i}
$$

MLP learn through an iterative process of adjustments applied to their free parameters. The most common learning algorithms are the standard back-propagation and fasterlearning variations. They use a gradient search technique to minimize a cost function equal to the mean square error (MSE) between the desired and the actual net outputs.

$$
M S E=\frac{1}{l} \sum_{i=1}^{l}\left(y_{i}-\hat{y}_{i}\right)^{2}
$$

The net is trained by initially selecting small random weights and internal thresholds, and presenting all training data repeatedly. Weights are adjusted after every trial using Information specifying the correct class until weights converges and the cost function is reduced to an acceptable value.
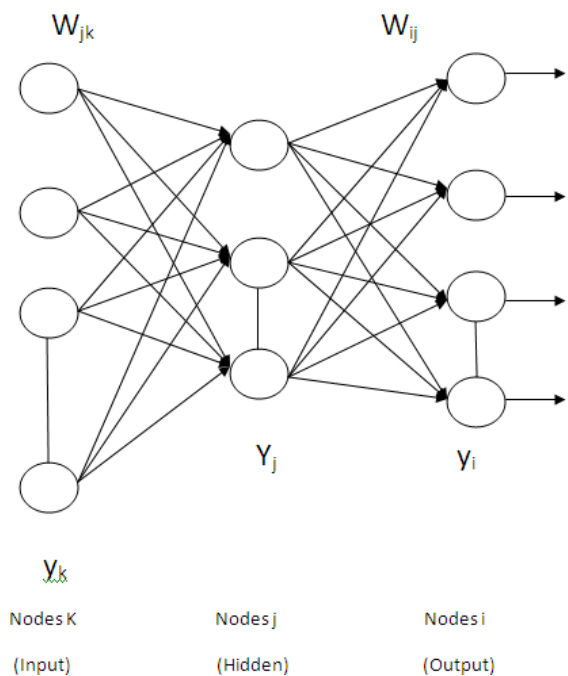

Typical Architecture of a MLP Neural Network System

\section{EXPERIMENT}

The learning process takes place through adjustment of weight connections between the input and hidden layers and between the hidden and output layers. The number of input nodes at the input layer was set at 17 according to the statistical features discussed in section II. Followings are the input nodes:

1. Mean Radius

2. Standard Deviation of Radii

3. Normalized Area

4. Perimeter

5. Mean of Max. Radii

6. Mean of Min Radii

The number of output nodes in this study was set at 6 as circle, half circle, equilateral triangle, square and hexagon. The number of nodes in the hidden layer was chosen based on trial and error. Each pattern presentation is tagged with its respective label as shown in below table. The maximum value in each row (0.9) identifies the corresponding node expected to secure the highest output for a pattern to be considered correctly classified. The output values are denoted as $\mathrm{O}_{1}, \mathrm{O}_{2}, \mathrm{O}_{3}, \mathrm{O}_{4}, \mathrm{O}_{5}$ and $\mathrm{O}_{6}$.

\begin{tabular}{|c|c|c|c|c|c|c|c|}
\hline Target & \multirow{2}{*}{ Des. } & \multicolumn{7}{|c|}{ Nodes } \\
\cline { 3 - 8 } Class & & 1 & 2 & 3 & 4 & 5 & 6 \\
\hline 1 & Square & 0.9 & 0.1 & 0.1 & 0.1 & 0.1 & 0.1 \\
\hline 2 & Circle & 0.1 & 0.9 & 0.1 & 0.1 & 0.1 & 0.1 \\
\hline 3 & $\begin{array}{c}\text { Half } \\
\text { Circle }\end{array}$ & 0.1 & 0.1 & 0.9 & 0.1 & 0.1 & 0.1 \\
\hline 4 & $\begin{array}{c}\text { Eq. } \\
\text { Triangle }\end{array}$ & 0.1 & 0.1 & 0.1 & 0.9 & 0.1 & 0.1 \\
\hline 5 & $\begin{array}{c}\text { Reg. } \\
\text { Hexagon }\end{array}$ & 0.1 & 0.1 & 0.1 & 0.1 & 0.9 & 0.1 \\
\hline 6 & $\begin{array}{c}\text { Reg. } \\
\text { Pentagon }\end{array}$ & 0.1 & 0.1 & 0.1 & 0.1 & 0.1 & 0.9 \\
\hline
\end{tabular}

The activation functions used were hyperbolic tangent for the hidden layer and sigmoid for the output layer. The hyperbolic tangent function is given by:

$$
f(x)=e^{x}-e^{-x} / e^{x}+e^{-x}
$$

The sigmoid function is given by:

$$
f(x)=1 / 1+e^{-x}
$$

The input node parameters are normalized to the level $[0$, 1]. 30 no. of patterns ( 5 from each category) data sets are used to train the NN with a target of MSE $<0.01$ as acceptable criteria for stopping of the training process.

\section{RESULTS}

The NN is trained using the ANN tool box in MATLAB. The set of statistical parameters used are extracted using the 
feature extraction process. Table-2 gives the set of parameters for the respective shapes. Following results are obtained after the training is complete and new test pattern data sets are provided for testing of the classifier:

\begin{tabular}{|l|c|c|}
\hline \multicolumn{1}{|c|}{ Target Class } & $\begin{array}{c}\text { Percentage Correct } \\
\text { Classification }\end{array}$ & MSE \\
\hline $1-$ Square & 96.78 & 0.0099 \\
\hline 2 - Circle & 97.45 & 0.0098 \\
\hline $3-$ Half Circle & 98.34 & 0.0101 \\
\hline $\begin{array}{l}\text { Triangle Eq. } \\
\text { Tr Reg. } \\
\text { Hexagon }\end{array}$ & 96.64 & 0.0090 \\
\hline $\begin{array}{l}\text { Pentagon } \\
\text { Peg. }\end{array}$ & 97.34 & 0.0092 \\
\hline
\end{tabular}

\section{Conclusion}

Pattern recognition can be done both in normal computers and neural networks. Computers use conventional arithmetic algorithms to detect whether the given pattern matches an existing one. It is a straight forward method. It will say either yes or no. It does not tolerate noisy patterns. On the other hand, neural networks can tolerate noise and, if trained properly, will respond correctly for unknown patterns. Neural networks may not perform miracles, but if constructed with the proper architecture and trained correctly with good data, they will give amazing results, not only in pattern recognition but also in other scientific and commercial applications.

\section{REFERENCES}

[1] A Versatile Machine Vision System for Complex Industrial Parts (IEEE Trans. On Computers, Sep. 1977, No. 9, Vol. C-27)

[2] Wani, M. A. and Pham, D. T., 1999, Efficient control chart pattern recognition through synergistic and distributed artificial neural networks. Proceedings of the Institution of Mechanical Engineers: Part B, 213, 157-169

[3] Anil k. Jain Fellow IEEE, Robert P.W Duin \& Jian chang Mao, Senior Member IEEE "IEEE Transactions on Pattern Analysis and Machine Intelligence VOL 22 No.1, January 2000

[4] Enrique Frias -Marrtinez, Angel Sanchez \& Jose Velez "Support Vector Machines Vs Multi-Layer Perceptrons for Efficient Off-Line Signature, BioMetry \& Artificial Vision Group

[5] Aki Vehtari and Juko Lampinen Bayesian MLP Neural Networks for Image Analysis" Laboratory Of Computational engineering, Helsinki University Of Technology, Finland 2000

[6] Yas Abbas Alsultanny, Musbah M. Aqel , 2002, Pattern recognition using multilayer neural-genetic Algorithm.. Elsevier Science B.V. Nuerocomputing 51(2003) 237-247

[7] Richard Bridge, "Computer Image Processing." Tessella support services PLC, Oxon, England, Issue V1.R2.M0, June, 2003

[8] Raul C. Mauresan "Pattern Recognition Using Pulse coupled Neural Neural Network \& Discrete Fourier Transforms "Personal Research

[9] NI Vision Based Automatic Optical Inspection (AOI), Proceedings of 2009 IEEE International Conference on Applied Superconductivity and Electromagnetic Devices Chengdu, China, September 25-27, 2009 
Table - 2 for Input Statistical Parameters

\begin{tabular}{|c|l|l|c|l|c|c|c|}
\hline S.No. & Parameters & Circle & Square & $\begin{array}{l}\text { Semi- } \\
\text { Circle }\end{array}$ & $\begin{array}{l}\text { Reg. } \\
\text { Hexagon }\end{array}$ & $\begin{array}{l}\text { Eq. } \\
\text { Triangle }\end{array}$ & $\begin{array}{l}\text { Reg. } \\
\text { Pentagon }\end{array}$ \\
\hline 1 & Perimeter & 6.28 & 7 & 6.49 & 6 & 7 & 6.13 \\
\hline 2 & Area & 3.14 & 1 & 0.12 & 3.14 & 2.8 & 3.20 \\
\hline 3 & Mean Radius & 1.00 & 1.0 & 1.00 & 1.0 & 1.0 & 1.00 \\
\hline 4 & Standard Deviation & 0.00 & 0.25 & 0.43 & 0.15 & 0.35 & 0.20 \\
\hline 5 & Figure Aspect & 1.00 & 1 & 0.56 & 1 & 1.50 & 1.00 \\
\hline 6 & Max Rl & 1.00 & 1.25 & 1.20 & 1.15 & 1.5 & 1.20 \\
\hline 7 & Max R2 & 1.00 & 1.25 & 1.20 & 1.15 & 1.5 & 1.20 \\
\hline 8 & Max R3 & 1.00 & 1.25 & 1.47 & 1.15 & 1.4 & 1.12 \\
\hline 9 & Max R4 & 1.00 & 1.25 & 1.46 & 1.15 & 1.4 & 1.12 \\
\hline 10 & Mean of Max R & 1.00 & 1.25 & 1.33 & 1.15 & 1.45 & 1.15 \\
\hline 11 & Min R1 & 1.00 & 0.85 & 0.74 & 0.90 & 0.7 & 0.90 \\
\hline 12 & Min R2 & 1.00 & 0.85 & 0.74 & 0.90 & 0.7 & 0.89 \\
\hline 13 & Min R3 & 1.00 & 0.85 & 0.59 & 0.90 & 0.8 & 0.86 \\
\hline 14 & Min R4 & 1.00 & 0.85 & 0.59 & 0.90 & 0.8 & 0.86 \\
\hline 15 & Mean of Min R & 1.00 & 0.85 & 0.67 & 0.90 & 0.75 & 0.88 \\
\hline 16 & Intercept on +ve X-Axis & 1.00 & 0.90 & 1.20 & 1.0 & 0.8 & 1.02 \\
\hline 17 & Intercept on -ve X-Axis & 1.00 & 0.90 & 1.20 & 1.0 & 0.8 & 1.02 \\
\hline 18 & Intercept on +ve Y-Axis & 1.00 & 0.90 & 0.76 & 1.0 & 1.5 & 1.06 \\
\hline 19 & Intercept on -ve Y-Axis & 1.00 & 0.90 & 0.59 & 1.0 & 0.8 & 0.86 \\
\hline
\end{tabular}

